\title{
HD 143418: an unusual light variable and a double-lined spectroscopic binary with a CP primary ${ }^{\star \star \star}$
}

\author{
H. Božić ${ }^{1}$, M. Wolf ${ }^{2}$, P. Harmanec ${ }^{2,3}$, A. Prša ${ }^{4}$, J. R. Percy ${ }^{5}$, D. Ruždjak ${ }^{1}$, D. Sudar ${ }^{1}$, M. Šlechta ${ }^{3}$, \\ H. $\mathrm{Ak}^{6}$, and P. Eenens
}

\author{
1 Hvar Observatory, Faculty of Geodesy, Zagreb University, 10000 Zagreb, Croatia \\ e-mail: hbozic@geodet.geof.hr \\ 2 Astronomical Institute of the Charles University, V Holešovičkách 2, 18000 Praha 8, Czech Republic \\ Astronomical Institute, Academy of Sciences, 25165 Ondřejov, Czech Republic \\ 4 Department of Physics, University of Ljubljana, Jadranska 19, 1000 Ljubljana, Slovenia \\ 5 Erindale Campus and Department of Astronomy and Astrophysics, University of Toronto, Mississauga, ON L5L IC6, Canada \\ ${ }^{6}$ Department of Astronomy and Space Sciences, Faculty of Arts and Sciences, Erciyes University, 38039 Kayseri, Turkey \\ 7 Departamento de Astronomia, Universidad de Guanajuato, Apartado 144, 36000 Guanajuato, GTO, Mexico
}

Received 15 December 2004 / Accepted 9 November 2006

\section{ABSTRACT}

\begin{abstract}
Aims. After a serendipitious discovery that HD 143418 is a light variable, we analyzed numerous $U B V$ observations and Hipparcos $H_{\mathrm{p}}$ magnitudes transformed to Johnson $V$ to find out whether the object is a very unusual spectroscopic binary.

Methods. Initial reductions of new photometry were carried out with the HEC22 program, while the new spectra were reduced in the IRAF and SPEFO programs. Orbital elements were derived with the FOTEL program, the KOREL disentangling was applied, and period searches were carried out using phase dispersion minimalization technique and the program PERIOD04. Final modeling of radial-velocity and light variations was carried out in PHOEBE.

Results. The brightness of HD 143418 varies with a period of $2.282520 \pm 0.000010$ and with little color changes. The light curve has a non-sinusoidal shape, which moreover changes from season to season. In each season, it can be described by the basic frequency and its first harmonic. Its long-term variations are characterized by cyclic variations of the amplitudes of the two frequencies and also the phase of the first frequency, the phase of the first harmonic remaining secularly constant. The amplitude of the principal frequency varies over a larger range and in antiphase to that of the first harmonic. Radial-velocity variations with the same period identify the object as a double-lined spectroscopic binary. The secondary spectrum is very faint, however. The rotation of the CP primary is strongly subsynchronous (its probable rotation period is $6.8 \pm 0.2$ ). We show that the observed light variations can be understood as a combination of the ellipsoidal variability in the binary system and either a secularly varying pattern of spots on the secondary or an inhomogeneous corotating cloud ejected from the primary.

Conclusions. Obviously, HD 143418 is an astrophysically interesting and dynamically unusual system that deserves future systematic spectral and photometric monitoring and theoretical modeling.
\end{abstract}

Key words. binaries: spectroscopic - stars: individual: HD 143418- stars: chemically peculiar

\section{Introduction}

For many years, the star HD 143418 (BD+40999, HIP 78226; $V=7.45)$ served as a recommended check star for observations of the Be variable V439 Her $=4$ Her, secured within the framework of the International Campaign for Photometric Monitoring of Bright Northern Be stars by Harmanec et al. (1980, 1982). Later it was also used as a transformation standard in the $U B V$ photometric programs - see, e.g., Harmanec et al. (1994) or Schuster et al. (1985). Using low-dispersion prismatic spectra, Bartaya (1979) classified HD 143418 as an A3m: star. Some uvby observations were published by Alania et al. (1989), but there is no report of light variability of this star either in the electronic version of the Moscow General Catalogue of Variable Stars or in the Strasbourg CDS bibliography. While reducing our

^ Based on spectral and photometric observations from the following observatories: Hvar, Ondřejov, San Pedro Mártir, Tubitak, and Phoenix. $\star \star$ Table 3 is only available in electronic form at the CDS via anonymous ftp to cdsarc.u-strasbg.fr $(130.79 .128 .5)$ or via http://cdsweb.u-strasbg.fr/cgi-bin/qcat?J/A+A/464/263 accurate $U B V$ observations secured in April 2001 at San Pedro Mártir Observatory (SPM hereafter) and at Hvar, we noted large deviations of this star in the all-sky photometry on several nights. Further monitoring and also investigation of Hvar $U B V$ data archives (Harmanec et al. 1997b) and Hipparcos $H_{\mathrm{p}}$ observations (Perryman \& ESA 1997) convinced us that the star is a smallamplitude light variable. Subsequently, we obtained 13 CCD spectra of HD 143418 at the coudé focus of the Ondřejov 2-m reflector to find out that the object is also a double-lined spectroscopic binary. Here, we report the results of our analyses.

\section{Observations and reductions}

\subsection{Photometry}

Photoelectric photometry suitable for the analysis of the light variations of HD 143418 consists of the following six data sets:

1. Systematic $U B V$ observations from Hvar Observatory, Croatia, secured from 1982 to 2005 with the 0.65 -m reflector. Observations obtained before 1991 were reduced and transformed into the standard $U B V$ system with the help of the 
HEC22 release 12 reduction program via nonlinear transformation formulæ (see Harmanec et al. 1994; and Harmanec \& Horn 1998) and have been already published by Pavlovski et al. (1997) and Harmanec et al. (1997b). More recent observations, obtained from 1993 to 2005, were reduced with the improved release 14.1 of the HEC22 program, which also permits us to model a time-variable extinction during observing nights.

2. $U B V$ observations obtained with the $0.84-\mathrm{m}$ reflector and Cuenta-pulsos photometer of SPM Observatory, Mexico, between 2001 and 2003. All measurements were reduced and transformed into the standard $U B V$ system via nonlinear transformation formulæ also using the latest release 14.1 of the HEC22 program.

3. $U B V$ observations secured with the $0.40-\mathrm{m}$ reflector of the Tubitak Observatory, Turkey in 2003, which were also reduced and transformed into the standard $U B V$ system using HEC22 rel.14.1

4. $U B V$ observations secured with the 0.25-m Automatic Photometric Telescope (APT) in Phoenix, Arizona (Percy \& Attard 1992; Percy et al. 1997). These measurements were reduced via linear transformation formulæ into the standard $U B V$ system by the APT service, a non-profit service directed by Professor Michael Seeds.

5. $U B V$ observations secured with the $0.65-\mathrm{m}$ reflector of the Ondřejov Observatory, Czech Republic, between 1983 and 1984, which were reduced and transformed into the standard $U B V$ system via nonlinear transformation formulæ using HEC22 rel. 12.

6. Broad-band all-sky $H_{\mathrm{p}}$ magnitude observations secured by the Hipparcos satellite (Perryman \& ESA 1997) between 1989 and 1993 and transformed to the standard Johnson $V$ with the help of a transformation formula derived by Harmanec (1998).

All data from ground-based stations were carefully examined for the quality of observations and their homogeneity; we include in our analysis the differential measurements relative to the comparison star $v$ Her (HD 144206) only. Such an approach enabled us to reduce the possible systematic difference between zero points of various observatories to a high degree. Also, allsky Hipparcos observations with error flags larger than 1 were excluded from our final data set.

For the comparison star $v$ Her the following all-sky values

$V=4^{\mathrm{m}} .739, \quad B-V=-0^{\mathrm{m}} \cdot 096, \quad U-B=-0.326$,

based on homogenization of numerous all-sky observations from Hvar and SPM observatories, have been adopted and then added to the magnitude differences HD $143418-v$ Her from all stations. The A-type $\operatorname{star} \varphi$ Her

$V=4^{\mathrm{m}} \cdot 425, \quad B-V=-0.064, \quad U-B=-0.249$

served as a check star for most of the ground-based observations and was observed as frequently as the variable and reduced in the same way. This star is a HgMn-type CP star and 0. 015 light variations in the $U$-band with a 7.832 period were reported by Rakosch \& Fiedler (1978). Photometric observations by Zboril $\&$ Budaj (1999) did not confirm the variability of the star, Winzer (1974) found no variations in $U, B$, and $V$ that would be greater than 0.007, and Adelman's analysis of the Hipparcos photometry also indicated its constancy (Adelman 1998). Our measurements over many seasons do not show any significant variability (see the bottom panels of Figs. 2 and 4).
Table 1. Journal of photoelectric observations; observing stations and instruments are identified by their running numbers from the Ondřejov/Praha photometric archives as follows:

1... Hvar Observatory, Croatia; 4... Ondřejov Observatory, Czech Republic; 15... APT-10, Phoenix, Arizona, USA; 30... San Pedro Mártir, Mexico; 61... Hipparcos satellite; 66... Tubitak, Turkey.

\begin{tabular}{rcrcc}
\hline \hline $\begin{array}{r}\text { Station } \\
\text { No. }\end{array}$ & $\begin{array}{c}\text { Epoch } \\
(\text { HJD-2 400 000) }\end{array}$ & $\begin{array}{r}\text { No. of } \\
\text { obs. }\end{array}$ & Passbands & Remark \\
\hline 1 & $45117.4-53535.4$ & 291 & $U B V$ & differential \\
4 & $45461.5-45912.4$ & 12 & $U B V$ & " \\
61 & $47864.6-49057.1$ & 111 & $B V$ & all-sky \\
15 & $48044.7-48440.7$ & $*$ & $U B V$ & differential \\
30 & $52059.7-52752.8$ & 79 & $U B V$ & " \\
66 & $52760.3-52769.4$ & 44 & $U B V$ & $"$ \\
\hline
\end{tabular}

* Different numbers of observations were obtained in different passbands: 37 in $U, 38$ in $B$, and 40 in $V$.

Basic information about all photometric observations is in Table 1 . To provide some idea about the quality of the transformation of $U B V$ data from individual stations into the standard system, we tabulate the seasonal mean differential $U B V$ values of the check star HD 145389 for individual stations in Table 2. The individual differential $U B V$ observations of HD 143418 are given in Table 3 .

\subsection{Spectroscopy}

Thirteen CCD spectra of HD 143418 covering the $\mathrm{H} \alpha$ region and two around $\mathrm{H} \beta$ were secured with a $C C D$ detector attached to the medium 0.7-m camera at the coude focus of the $2 \mathrm{~m}$ telescope of the Ondřejov Observatory, Czech Republic. The spectra were obtained between March 2004 and May 2005 and have linear dispersions of $8.5 \AA \mathrm{mm}^{-1}$ (blue) and $17.2 \AA \mathrm{mm}^{-1}$ (red) and a 2-pixel resolving power of about 12600 . Their $S / N$ ratios are between 206 and 497, with the exception of the underexposed spectrum taken on JD 2453145.5313, which has the $S / N$ ratio of only 79 .

The initial reduction of the spectra (bias subtraction, flatfielding, wavelength calibration, and creation of 1-D images) was carried out by MŠ in IRAF. The rectification of the spectra and the radial-velocity (RV hereafter) and line-intensity measurements were carried out by PH in SPEFO (Horn et al. 1996; Škoda 1996). The SPEFO program enables users to carry out complete reductions of electronic or digitized photographic spectra entering as 1-D images (wavelength and intensity calibration, continuum rectification, interactive removal of cosmic spikes, RV, central line intensity, and equivalent-width measurements). In particular, during the RV measurement, SPEFO displays the direct and flipped line profiles on the computer screen. This allows the user to measure specific parts of line profiles (outer wings, line cores, and so on) by shifting the flipped profile over the direct one until the desired overlap of the respective parts of the profile is achieved. The journal of spectroscopic observations is given in Table 4 together with the mean RVs measured for both binary components - see Sect. 4.2 for details.

\section{Period analysis of the photometric data}

Inspection of Table 3 reveals that, with the exception of the APT measurements, there are 3 to 5 observations per night in sequences that predominantly did not last longer than 0.055 days. 
Table 2. Mean differential $U B V$ values of the check star $\varphi$ Her (HD 145389) relative to $v$ Her from individual stations and observing seasons, illustrating the level of homogeneity of our transformations to the standard system. The observing stations are identified by the same running numbers as in Table 1.

\begin{tabular}{rcrrccc}
\hline \hline $\begin{array}{r}\text { Station } \\
\text { No. }\end{array}$ & $\begin{array}{c}\text { Mean epoch } \\
\text { (HJD-2 400 000) }\end{array}$ & Year & $\begin{array}{r}\text { No. of } \\
\text { obs. }\end{array}$ & $V$ & $B-V$ & $U-B$ \\
\hline 1 & 45072.6 & 1982 & 99 & $4.252 \pm 0.008$ & $-0.064 \pm 0.008$ & $-0.248 \pm 0.011$ \\
1 & 51377.2 & 1999 & 5 & $4.257 \pm 0.007$ & $-0.062 \pm 0.009$ & $-0.248 \pm 0.013$ \\
1 & 52305.2 & 2002 & 139 & $4.254 \pm 0.006$ & $-0.062 \pm 0.008$ & $-0.252 \pm 0.008$ \\
1 & 52691.4 & 2003 & 42 & $4.255 \pm 0.007$ & $-0.061 \pm 0.010$ & $-0.255 \pm 0.010$ \\
1 & 53039.0 & 2004 & 96 & $4.254 \pm 0.005$ & $-0.061 \pm 0.005$ & $-0.253 \pm 0.006$ \\
1 & 53370.2 & 2005 & 78 & $4.257 \pm 0.006$ & $-0.064 \pm 0.006$ & $-0.252 \pm 0.006$ \\
\hline 30 & 52608.7 & 2002 & 48 & $4.259 \pm 0.007$ & $-0.062 \pm 0.007$ & $-0.247 \pm 0.007$ \\
30 & 52750.7 & 2003 & 28 & $4.257 \pm 0.006$ & $-0.061 \pm 0.007$ & $-0.249 \pm 0.005$ \\
\hline 61 & 48078.1 & 1990 & 43 & $4.248 \pm 0.004$ & all-sky & \\
61 & 48377.8 & 1991 & 45 & $4.249 \pm 0.005$ & all-sky & \\
61 & 48700.0 & 1992 & 24 & $4.249 \pm 0.004$ & all-sky & \\
61 & 49046.7 & 1993 & 6 & $4.246 \pm 0.007$ & all-sky & \\
\hline 66 & 52764.7 & 2003 & 35 & $4.253 \pm 0.007$ & $-0.062 \pm 0.006$ & $-0.244 \pm 0.009$ \\
\hline
\end{tabular}

Table 4. Journal of spectroscopic observations

\begin{tabular}{cccrc}
\hline \hline File & $\begin{array}{c}\text { Mid-exposure } \\
(\text { HJD-2 400000) }\end{array}$ & $\begin{array}{c}\text { Spectral } \\
\text { range }(\AA)\end{array}$ & $\begin{array}{c}R V_{1} \\
\left(\mathrm{~km} \mathrm{~s}^{-1}\right)\end{array}$ & $\begin{array}{c}R V_{2} \\
\left(\mathrm{~km} \mathrm{~s}^{-1}\right)\end{array}$ \\
\hline nc310051 & 53096.4806 & $4701-4953$ & 3.2 & - \\
nd010037 & 53097.4663 & $6255-6767$ & -8.0 & - \\
nd010055 & 53097.5291 & $4780-5031$ & -19.8 & - \\
nd070031 & 53103.4231 & $6255-6767$ & 24.5 & -106.1 \\
nd270011 & 53123.4953 & $6255-6767$ & -74.3 & 70.5 \\
nd290022 & 53125.3412 & $6255-6767$ & -93.5 & 88.2 \\
nd290024 & 53125.3851 & $6255-6767$ & -96.2 & 92.5 \\
ne190052 & 53145.5313 & $6255-6767$ & -35.1 & - \\
ne190053 & 53145.5427 & $6255-6767$ & -38.6 & 47.2 \\
oe110019 & 53502.4662 & $6255-6767$ & -64.3 & 56.6 \\
oe130022 & 53504.3674 & $6255-6767$ & -101.8 & 93.9 \\
oe190008 & 53510.3875 & $6255-6767$ & 31.7 & -110.6 \\
oe190012 & 53510.4823 & $6255-6767$ & 13.8 & -104.7 \\
\hline
\end{tabular}

To achieve a more thorough analysis, the time span of several nights reached a quarter of the day. Keeping in mind that the A-type star may exhibit $\delta$-Scuti-type variations with a period as short as $1 \mathrm{~h}$, we first performed a frequency analysis of individual measurements. We analyzed the most numerous set of $V$-band observations over the range from 0 to $30 \mathrm{~cd}^{-1}$ using the discrete Fourier transform (DFT; Deeming 1975). The power spectrum depicted in Fig. 1 shows the highest peak for the frequency $f=0.438 \mathrm{c} \mathrm{d}^{-1}$, the $1-f=0.562 \mathrm{c} \mathrm{d}^{-1}$ reflection, the if $=0.876 \mathrm{c} \mathrm{d}^{-1}$ alias, and also a series of harmonics. There is no evidence of any prominent frequencies in the high frequency range. After verifying that HD 143418 does not vary significantly on time scales shorter than $1 \mathrm{~h}$, we created 0.055 -day normals of all data to improve the $S / N$ ratio. Analysis of the $V$ band normals was carried out for the frequency range from 0 to $1 \mathrm{c} \mathrm{d}^{-1}$ and, again, the most prominent peak emerges for the frequency $f=0.438 \mathrm{~cd}^{-1}$. Figure 2 shows the power spectrum of HD 143418 (upper panel) and, for comparison, the power spectrum of the check star $\varphi$ Her (bottom panel). A trial phase diagram for the best period showed clear variations with a doublewave light curve.

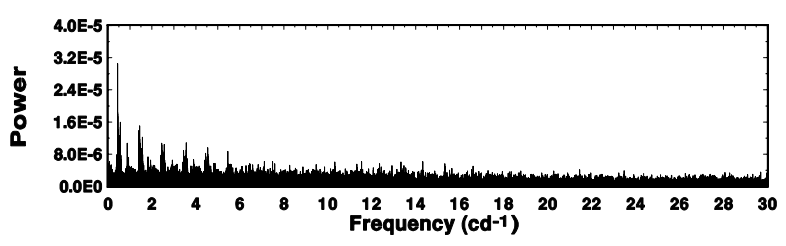

Fig. 1. Power spectrum of the individual $V$ measurements for HD 143418 in the frequency range from 0 to 30 cycle/day.

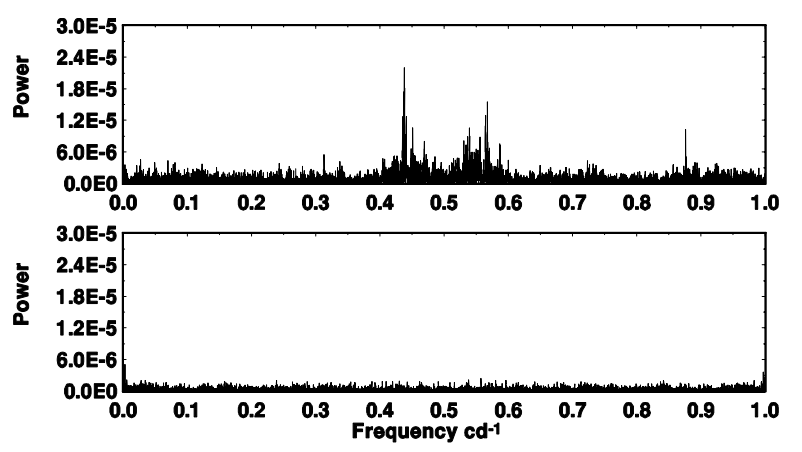

Fig. 2. Power spectrum of the 0.055 day means of $V$ observations for HD 143418 (upper panel) and the check star $\varphi$ Her (bottom panel).

To this end, we applied the non-linear least-squares fit to the $V$-magnitude 0.055 -day normals (considering their relative weights) using the PERIOD04 program (Lenz \& Breger 2005) and allowing for the convergence of the main frequency and its harmonics. We found that the rms error of the fit was improved significantly only after the inclusion of the first harmonic, the higher ones having negligibly small amplitudes and leading to only a marginal decrease of the rms of the fit. We therefore derived the final fits taking into account the main frequency and its first harmonic only. The period obtained this way for all (the most numerous and most accurate) $V$ observations listed in Table 1, which we adopt in the rest of this paper, is $P=2$ d.282520 \pm 0.0 .000010 . As already mentioned earlier and discussed in detail in Sect. 4.2 below, we found that the observed A star is actually the primary component of a binary system with the orbital period identical (or very similar) to the period of photometric changes and a circular orbit. To make various phase 


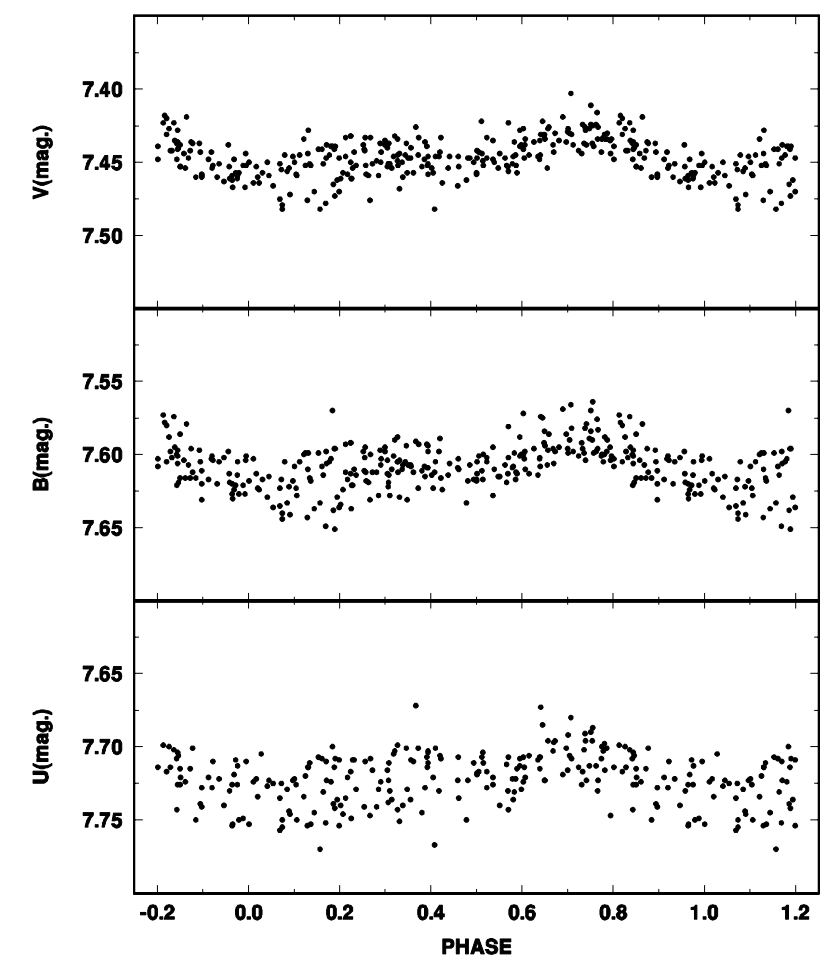

Fig. 3. $U B V$ light curves of HD 143418 plotted for ephemeris (3).

plots presented here directly comparable, from here on we therefore adopt the final ephemeris, based on the PHOEBE combined solution of RV and light curves (see Sect. 5.3 and Table 7 below), adopting the epoch of the superior conjunction of the primary as phase zero:

$T_{\text {super.conj. }}=$ HJD $2449178.43247(99)+2.282520(10) \times E$.

The $U B V$ light curves phased with ephemeris (3) are shown in Fig. 3. One can see that they deviate strongly from a sinusoidal shape and are quite reminiscent of the light curves of some chemically peculiar (CP) stars like 46 Eri $=$ HR 1449 (Manfroid \& Renson 1989) or PV And = HD 224166 (Stagg et al. 1988; Harmanec et al. 1991), another originally recommended check star for the Be observing campaign which turned out to be variable. The large scatter of data at a given phase, evident in light curves depicted in Fig. 3, led us to suspect that the light-curve shape might be secularly variable. To verify this, we investigated light curves for the seasonal subsets of data (or subsets covering up to about 500 days when not enough observations during one season were available). The phase plots for 8 seasons (cf. Table 5) are shown in Fig. 4, where the corresponding plots for the check star $\varphi$ Her are also included. Their inspection reveals several interesting facts:

1. There is little doubt that the light curve is indeed secularly variable.

2. During the first four seasons, the light curve has two similar light minima and maxima.

3. In the fifth season (HJD $2451372-2452$ 172), the amplitude of the light curve has increased and its shape has changed quite dramatically to a single-wave curve with a sharp maximum. This explains why we discovered the light variations just at that time.

4. In the next season, the light curve changed again, having a flat maximum and one sharp minimum.

5. The obvious constancy of the check star $\varphi$ Her in all seasons safely excludes the possibility that the variations of
HD 143418 would be affected by some small changes of the comparison star or other systematic effects. The constancy of the comparison star $v$ Her is also confirmed by its Hipparcos all-sky photometry (not shown here). Note also that the light curve from the time interval HJD $2448343-49057$ (S4) contains observations with the $0.25-\mathrm{m}$ Automatic Telescope. The smaller aperture of the telescope and the fact that this data set was transformed to the standard system only via linear transformation formulæ may explain a somewhat larger scatter of the curve ${ }^{1}$.

To quantify these findings, we again applied the PERIOD04 program to derive the fits to the (most accurate) $V$ light curves for all subsets. The results are shown in Table 5 and the secular variations of the amplitudes and phases of the main frequency and its first harmonic are shown graphically in Fig. 5. Inspecting Fig. 5, one arrives at the following conclusions:

1. The amplitude of the principal frequency $f_{1}$ undergoes a smooth and possibly cyclic (if not periodic) variation with a cycle length of some 9000 days, while the amplitude of the first harmonic varies in antiphase to that and with a much smaller a mplitude.

2. The phase of the principal frequency undergoes a secular cyclic variation in antiphase with its amplitude variation, while the phase of the first harmonic seems to remain more or less constant.

\section{Spectral analysis of HD 143418}

\subsection{Comparison of line spectra with a synthetic spectrum}

To obtain some estimate of the effective temperature and local gravity of the primary, we used synthetic spectra calculated from the Kurucz (1994) grid of solar composition LTE line blanketed model atmospheres with the help of the program SYNSPEC - see Hubeny et al. (1985) ${ }^{2}$. Oscillator strengths, wavelengths, and damping parameters for all lines contributing to the resulting spectrum in the neighborhood of the line profiles in question were taken from the list of Kurucz (1993). The Stark broadening of hydrogen Balmer lines was calculated using the tables of Vidal et al. (1973). The synthetic spectra from SYNSPEC were then rotationally broadened in SPEFO.

After several trials, we found that both the red and blue spectra can best be fitted with a Kurucz (1994) model for $T_{\text {eff }}=$ $7700 \mathrm{~K}$ and $\log g=4.0$ [cgs], which corresponds to an A7 star. The best fit was achieved for the projected rotational velocity $v \sin i=18 \mathrm{~km} \mathrm{~s}^{-1}$. A comparison of the observed blue spectrum with the model spectrum is shown in Fig. 6. It is obvious that the strengths of certain metallic lines are quite different in the observed and model spectra. Varying effective temperature and gravity over the range of temperatures corresponding to A stars, we were unable to remove this discrepancy. Two enlarged parts of the same spectrum are shown in Figs. 7 and 8. It is clearly seen from Fig. 7 that the CaII lines are highly underabundant, which is a typical property of Am stars but less so of Ap stars. The overabundancy of the CrII lines displayed in Fig. 8 is a characteristic

\footnotetext{
1 As discussed in detail in Harmanec et al. (1994), the non-linearity of the transformation occurs around $B-V \sim 0$, which implies that when the differential photometry of an A star is obtained relative to a B star comparison or vice versa, the linear tranformation may introduce larger errors.

2 For the most recent update of the program and its manual, see Hubeny \& Lanz (2000), http: //tlusty.gsfc .nasa.gov
} 


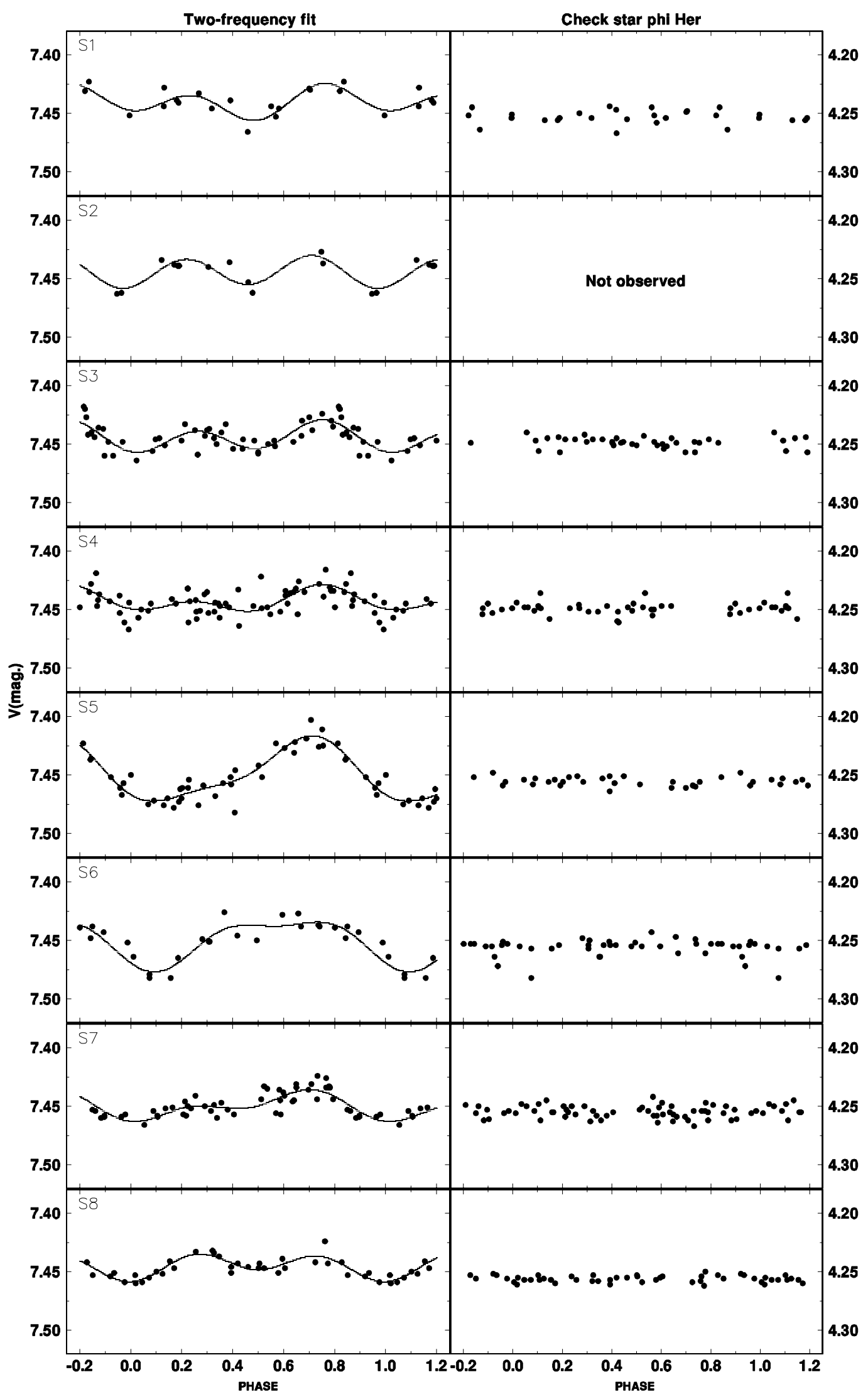

Fig. 4. Observed (0.055-d normals shown as dots) and calculated (solid lines) $V$ light changes of HD 143418 plotted vs. phase from ephemeris (3) and shown for data subsets spanning no more than about 500 days. The model curves are based on the respective two-frequency fits of Table 5 . The corresponding plots for the check star $\varphi$ Her are also shown (with the exception of one season when this star was not observed). The rms errors of the one observation of unit weight are all smaller than 0.019 , their mean value being 0.0028 . 
Table 5. Fits of $V$ light curves for the eight subsets of observations for the finally adopted frequency $f_{1}=0.438112 \mathrm{~cd}^{-1}$ and its first harmonic $f_{2}=0.876224 \mathrm{c} \mathrm{d}^{-1}$ derived with the help of the PERIOD04 program. The fit function is $V_{\text {calc }}^{i}(t)=L^{i}+A_{1}^{i} \cos \left(f_{1} t+2 \pi \delta_{1}^{i}\right)+A_{2}^{i} \cos \left(f_{2} t+2 \pi \delta_{2}^{i}\right)$, where $L^{i}$ is the level of the $i$-th season, $A_{1}^{i}$ and $A_{2}^{i}$ are the amplitudes, and $\delta_{1}^{i}$ and $\delta_{2}^{i}$ are phase shifts of the principal frequency and first harmonic waves, respectively. The rms error is the error of 1 observation of unit weight for the two-frequency fit. The errors of amplitudes and phases were estimated from the error matrix of the least-squares solution.

\begin{tabular}{|c|c|c|c|c|c|c|c|}
\hline Season & $\begin{array}{l}\text { HJD-2400000 } \\
\text { interval }\end{array}$ & $\begin{array}{l}\text { Freq. } \\
\left(\mathrm{cd}^{-1}\right)\end{array}$ & $\underset{\text { (mag) }}{\text { Amplitude }}$ & Phase & $\begin{array}{l}\text { Level } \\
\text { (mag) }\end{array}$ & $\begin{array}{c}T_{\max } \\
\text { HJD-2400000 }\end{array}$ & $\begin{array}{l}\mathrm{rms} \\
(\mathrm{mag})\end{array}$ \\
\hline 1 & $45117-45547$ & $\begin{array}{l}f_{1} \\
f_{2}\end{array}$ & $\begin{array}{l}0.0066 \pm 0.0019 \\
0.0108 \pm 0.0020\end{array}$ & $\begin{array}{l}0.6896 \pm 0.0528 \\
0.8537 \pm 0.0236\end{array}$ & 7.4408 & $\begin{array}{l}49178.1118 \\
49177.8555\end{array}$ & 0.0062 \\
\hline 2 & $45823-47006$ & $\begin{array}{l}f_{1} \\
f_{2}\end{array}$ & $\begin{array}{l}0.0022 \pm 0.0020 \\
0.0123 \pm 0.0018\end{array}$ & $\begin{array}{l}0.9586 \pm 0.0617 \\
0.9213 \pm 0.0173\end{array}$ & 7.4442 & $\begin{array}{l}49177.4979 \\
49177.7785\end{array}$ & 0.0053 \\
\hline 3 & $47864-48332$ & $\begin{array}{l}f_{1} \\
f_{2}\end{array}$ & $\begin{array}{l}0.0053 \pm 0.0013 \\
0.0105 \pm 0.0012\end{array}$ & $\begin{array}{l}0.8395 \pm 0.0355 \\
0.8356 \pm 0.0186\end{array}$ & 7.4447 & $\begin{array}{l}49177.7698 \\
49177.8763\end{array}$ & 0.0068 \\
\hline 4 & $48343-49057$ & $\begin{array}{l}f_{1} \\
f_{2}\end{array}$ & $\begin{array}{l}0.0073 \pm 0.0013 \\
0.0071 \pm 0.0014\end{array}$ & $\begin{array}{l}0.7891 \pm 0.0321 \\
0.8533 \pm 0.0300\end{array}$ & 7.4430 & $\begin{array}{l}49177.8851 \\
49177.8564\end{array}$ & 0.0088 \\
\hline 5 & $51372-52172$ & $\begin{array}{l}f_{1} \\
f_{2}\end{array}$ & $\begin{array}{l}0.0255 \pm 0.0007 \\
0.0074 \pm 0.0008\end{array}$ & $\begin{array}{l}0.8729 \pm 0.0060 \\
0.8642 \pm 0.0183\end{array}$ & 7.4483 & $\begin{array}{l}49177.6936 \\
49177.8436\end{array}$ & 0.0072 \\
\hline 6 & $52380-52477$ & $\begin{array}{l}f_{1} \\
f_{2}\end{array}$ & $\begin{array}{l}0.0198 \pm 0.0016 \\
0.0076 \pm 0.0014\end{array}$ & $\begin{array}{l}0.9503 \pm 0.0102 \\
0.6761 \pm 0.0354\end{array}$ & 7.4497 & $\begin{array}{l}49177.5169 \\
49178.0582\end{array}$ & 0.0074 \\
\hline 7 & $52740-52868$ & $\begin{array}{l}f_{1} \\
f_{2}\end{array}$ & $\begin{array}{l}0.0091 \pm 0.0008 \\
0.0065 \pm 0.0009\end{array}$ & $\begin{array}{l}0.9265 \pm 0.0149 \\
0.8913 \pm 0.0177\end{array}$ & 7.4499 & $\begin{array}{l}49177.5712 \\
49177.8127\end{array}$ & 0.0061 \\
\hline 8 & $53137-53535$ & $\begin{array}{l}f_{1} \\
f_{2}\end{array}$ & $\begin{array}{l}0.0053 \pm 0.0006 \\
0.0086 \pm 0.0007\end{array}$ & $\begin{array}{l}0.0779 \pm 0.0234 \\
0.8559 \pm 0.0117\end{array}$ & 7.4448 & $\begin{array}{l}49177.2256 \\
49177.8530\end{array}$ & 0.0046 \\
\hline
\end{tabular}

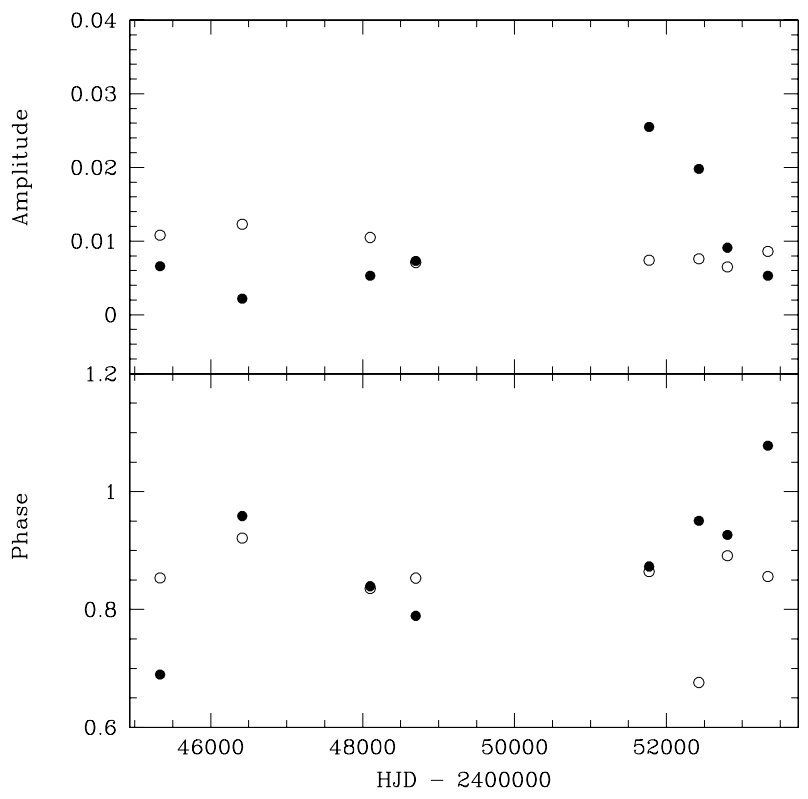

Fig. 5. Secular variations of the amplitudes and phases of the principal frequency $f_{1}$ (filled circles) and its first harmonic $f_{2}$ (open circles) with time.

of Ap as well as Am stars. As pointed out by the referee, the $g f$ values of some ions from Kurucz's line lists may not be optimal. For that reason, in Fig. 9, we also compare a blue spectrum of a normal A7 star 21 LMi obtained with the same Ondřejov spectrograph with a synthetic spectrum based on a Kurucz model. One can see a very reasonable agreement between the observed and synthetic spectra in this case, which in turn seems to confirm the Bartaya (1979) finding that HD 143418 is indeed a CP star.

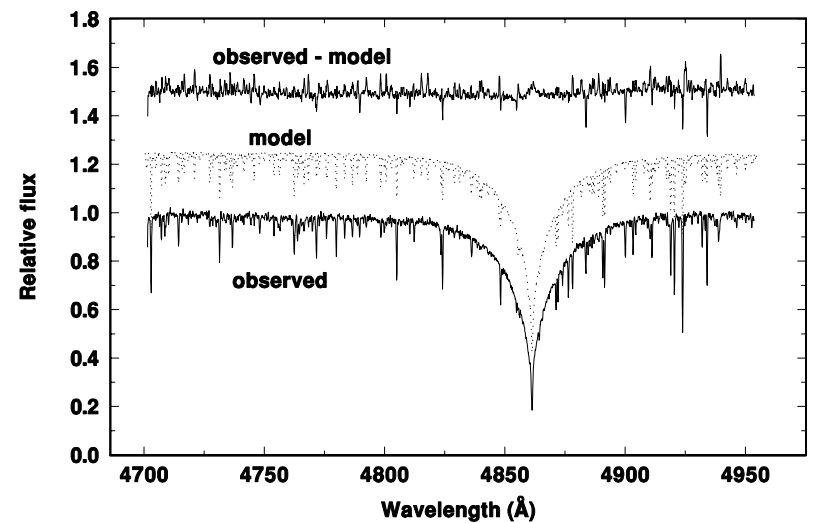

Fig. 6. The observed spectrum nc310051 in the neighborhood of $\mathrm{H} \beta$ from March 31, 2004 (full line) is compared to the solar composition Kurucz model for $T_{\text {eff }}=7700 \mathrm{~K}, \log g=4.0$ [cgs], and $v \sin i=$ $18 \mathrm{~km} \mathrm{~s}^{-1}$ (dotted line). The difference between the two is also shown in the upper part of the figure.

We stop here and leave a detailed study of the character of the chemical peculiarity of HD 143418 to the experts in the field.

We also measured and analyzed central intensities and equivalent widths of several well-resolved metallic lines in all available red spectra of the star. Although we cannot exclude some mild variations of line strength, we found no obvious dependence of these quantities on the phase of the 2.28 period. The number of observations is so limited that a meaningful period search is impossible until more spectra are accumulated. 


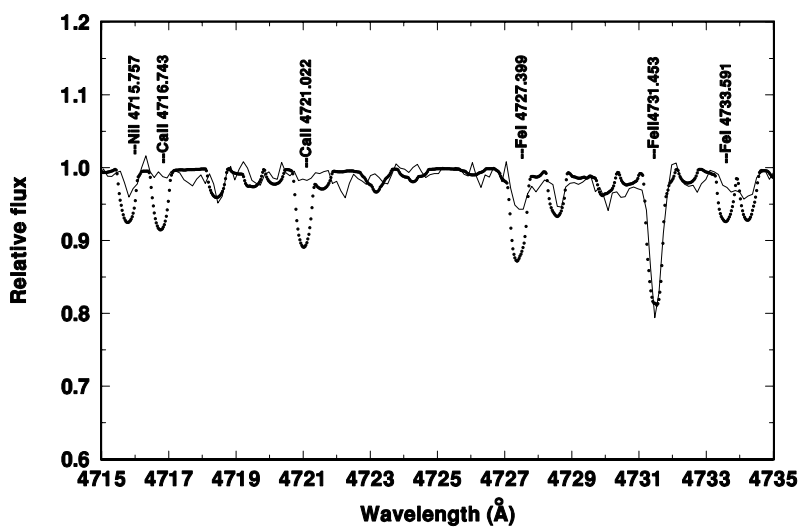

Fig. 7. An enlarged part of the observed spectrum nc310051 (thin line) compared to the synthetic spectrum (dotted line which appears as a thick line in some parts of the continuum where the density of data points is high) for the Kurucz (1994) model for $T_{\text {eff }}=7700 \mathrm{~K}$ and $\log g=4.0$ [cgs].

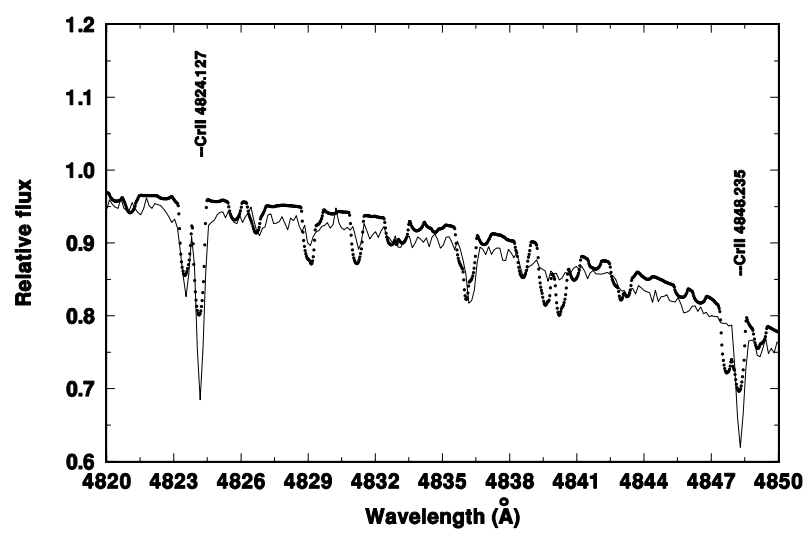

Fig. 8. Another enlarged part of the observed spectrum nc310051 (thin line) showing metallic lines that are blended with the blue wing of $\mathrm{H} \beta$, compared to the synthetic spectrum (dotted line which appears as a thick line in some parts of the continuum where the density of data points is high) for the Kurucz (1994) model for $T_{\text {eff }}=7700 \mathrm{~K}$ and $\log g=4.0$ [cgs].

Table 6. Circular-orbit elements from the FOTEL solutions. The value of the orbital period was fixed at 2.282520, derived from photometry. The epoch of RV maximum $T_{\mathrm{RV} \text { max. }}$ is given in HJD-2453000

\begin{tabular}{rrr}
\hline \hline Element & Solution 1 & Solution 2 \\
\hline$T_{\mathrm{RV} \text { max. }}$ & $99.2310 \pm 0.0031$ & $99.225 \pm 0.014$ \\
$K_{1}\left(\mathrm{~km} \mathrm{~s}^{-1}\right)$ & $80.02 \pm 0.97$ & $80.2 \pm 1.2$ \\
$K_{2}\left(\mathrm{~km} \mathrm{~s}^{-1}\right)$ & - & $133.3 \pm 1.9$ \\
$\gamma_{1}\left(\mathrm{~km} \mathrm{~s}^{-1}\right)$ & $-19.77 \pm 0.57$ & $-19.5 \pm 1.0$ \\
$\gamma_{2}\left(\mathrm{~km} \mathrm{~s}^{-1}\right)$ & - & $-26.0 \pm 6.0$ \\
\hline
\end{tabular}

\subsection{Duplicity of $H D 143418$}

Table 4 shows that the RV of HD 143418 varied from -102 to $+32 \mathrm{~km} \mathrm{~s}^{-1}$ during our observations and that these variations were quite rapid: the $\mathrm{RV}$ changed from -8 to $-19.8 \mathrm{~km} \mathrm{~s}^{-1}$ within 2.5 h of observations on April 1/2, 2004 (HJD 2453097). A period search and a phase plot immediately indicated that in spite of a very limited number of observations, the star is obviously a spectroscopic binary having the orbital period identical or very similar to that of the light changes. We used program FOTEL (Hadrava 1990, 2004a) to derive initial circular-orbit

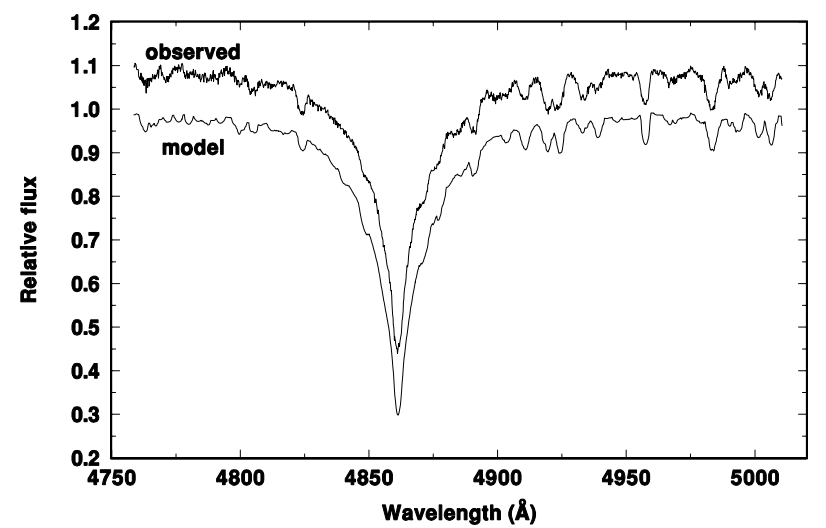

Fig. 9. A comparison of one observed spectrum of the A7V star $21 \mathrm{LMi}$ with a synthetic spectrum based on the Kurucz (1994) model for $T_{\text {eff }}=$ $8000 \mathrm{~K}$ and $\log g=4.0$ [cgs], rotationally broadened to $110 \mathrm{~km} \mathrm{~s}^{-1}$. The observed spectrum has beeen shifted for 0.2 in flux for a better clarity.

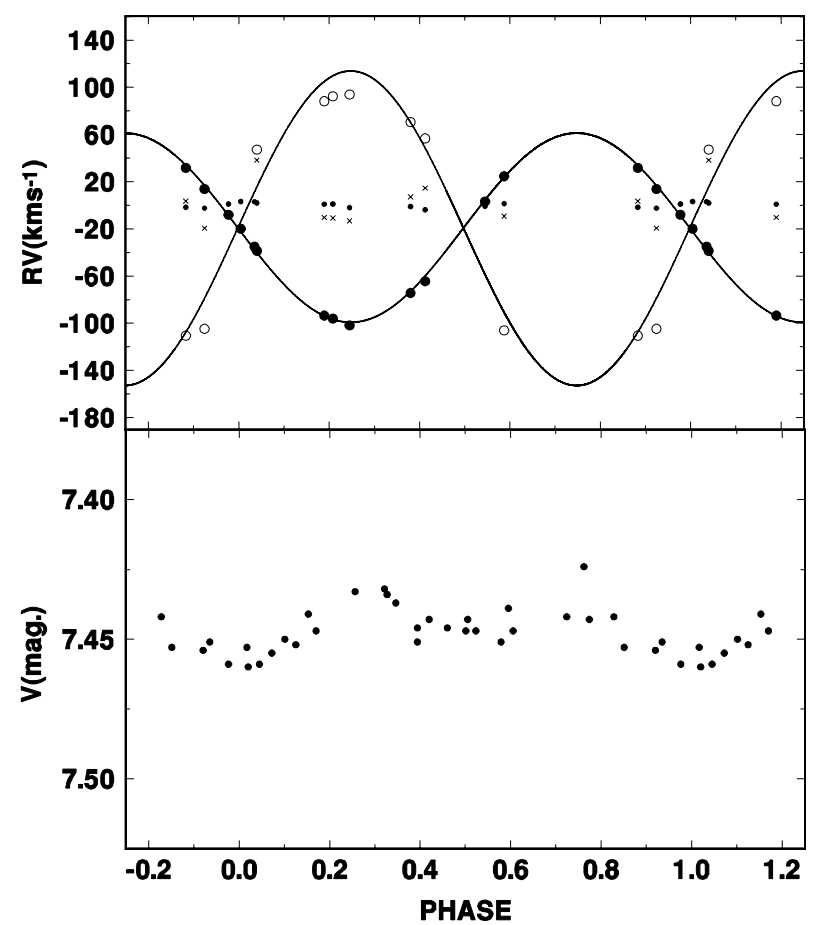

Fig. 10. Upper panel: RVs of the primary (filled circles) and secondary (open circles) components of $\mathrm{H} \delta$, based on the direct RV measurements given in Table 4, are plotted vs. phase from ephemeris (3). Small black dots and crosses show the $\mathrm{O}-\mathrm{C}$ deviations from solution 2 for the primary and secondary, respectively. Bottom panel: The $V$ light curve from season 8 shown to illustrate the mutual phase correspondence between the RV and light curves.

solutions. We adopted the period from ephemeris (3). A solution based on the mean RVs of the primary component for all 13 spectra (see Table 4) is given as solution 1 in Table 6.

Inspecting the $\mathrm{H} \alpha$ line profiles, we noted the presence of a weak $\mathrm{H} \alpha$ line from the secondary - see Fig. 11. To obtain an estimate of the semiamplitude of the secondary, we subtracted spectrum nd 010037, obtained close to the conjunction of both stars, properly shifted in RV, from all other red spectra. Then we measured the RV of the weak secondary in the residual spectra, omitting uncertain cases. These secondary RVs are also given in Table 4. Solution 2 in Table 6 is another FOTEL solution, in which these secondary RVs were also considered. One can see 


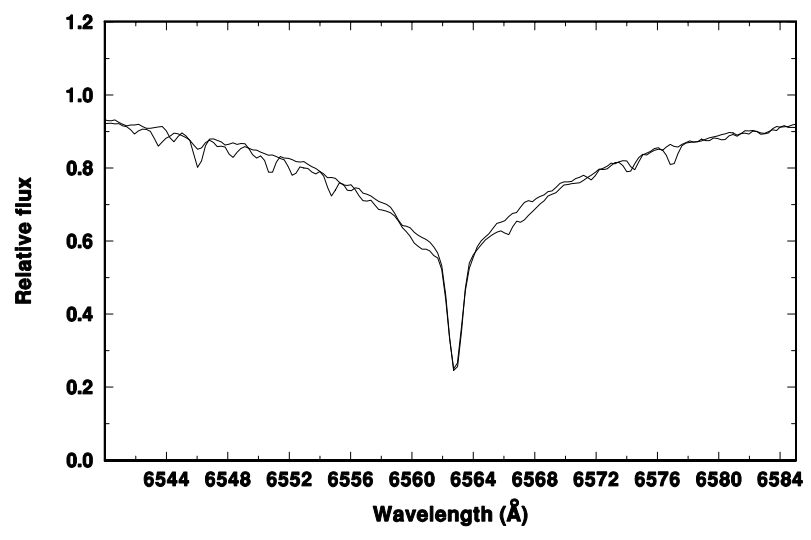

Fig. 11. A comparison of central parts of two $\mathrm{H} \alpha$ line profiles: The thicker line shows the spectrum nd070031 shifted by $-24.8 \mathrm{~km} \mathrm{~s}^{-1}$, the thinner line shows spectrum nd290024 shifted by $+96.1 \mathrm{~km} \mathrm{~s}^{-1}$ in radial velocity. One clearly sees a trace of a faint secondary component, blueshifted for the first, and red-shifted for the second spectrum (cf. Table 4).

that the systemic velocities for both components are identical within the limits of their respective errors, which increased the credibility of the result. The corresponding RV curves are shown in Fig. 10.

In the next step, we attempted to apply the disentangling program KOREL (Hadrava 1995, 1997, 2004b) to the red spectra. KOREL allows us to include the telluric lines into the solution. Readers are referred to Hadrava (1995, 2004b) for the description of this procedure, and to Harmanec et al. (1997a) for its first practical application. All individual spectra were weighted by the square of their $S / N$. Due to the very limited number of spectra, the solution in which allowance was made for a free convergence of all relevant orbital elements turned out to be unstable. We therefore followed the advice given by Dr. Hadrava: We kept the orbital period fixed at the value known from photometry, and first derived a solution for the primary and for telluric lines with variable line strengths for the wavelength interval from 6465 to $6510 \AA$ only. This particular region contains a number of strong telluric lines. Then, keeping the line strengths for the individual telluric spectra from this solution fixed, we used KOREL to disentangle the spectra of both binary components, keeping the orbital elements fixed from FOTEL solution 2. To achieve the best resolution in wavelength, we split the observed spectra into two intervals: one from 6272 to $6446 \AA$, which contains the region of the Si II 6347 and $6371 \AA$ doublet, and another one from 6450 to $6750 \AA$, which contains the $\mathrm{H} \alpha$ region and the weak He I 6678 line.

The disentangled spectra of both binary components are shown in Fig. 12. KOREL indeed detected a weak $\mathrm{H} \alpha$ line and a few very weak metallic lines of the secondary, which seem to correspond to a spectrum of some $5700-6000 \mathrm{~K}$. We also found that the projected rotational velocity of the secondary is some $35 \mathrm{~km} \mathrm{~s}^{-1}$. However, this value is subject to a relatively large uncertainty due to the weakness of the secondary spectrum.

\section{Binary properties and basic physical elements}

Once the preliminary results based on analyses of frequencies and spectra have been obtained, we proceed with full-fledged modeling. As Fig. 10 shows, the mutual phase correspondence of the RV and light changes for some seasons (S1-S4 and S8, which is shown in Fig. 10; compare also Fig. 4) is reminiscent
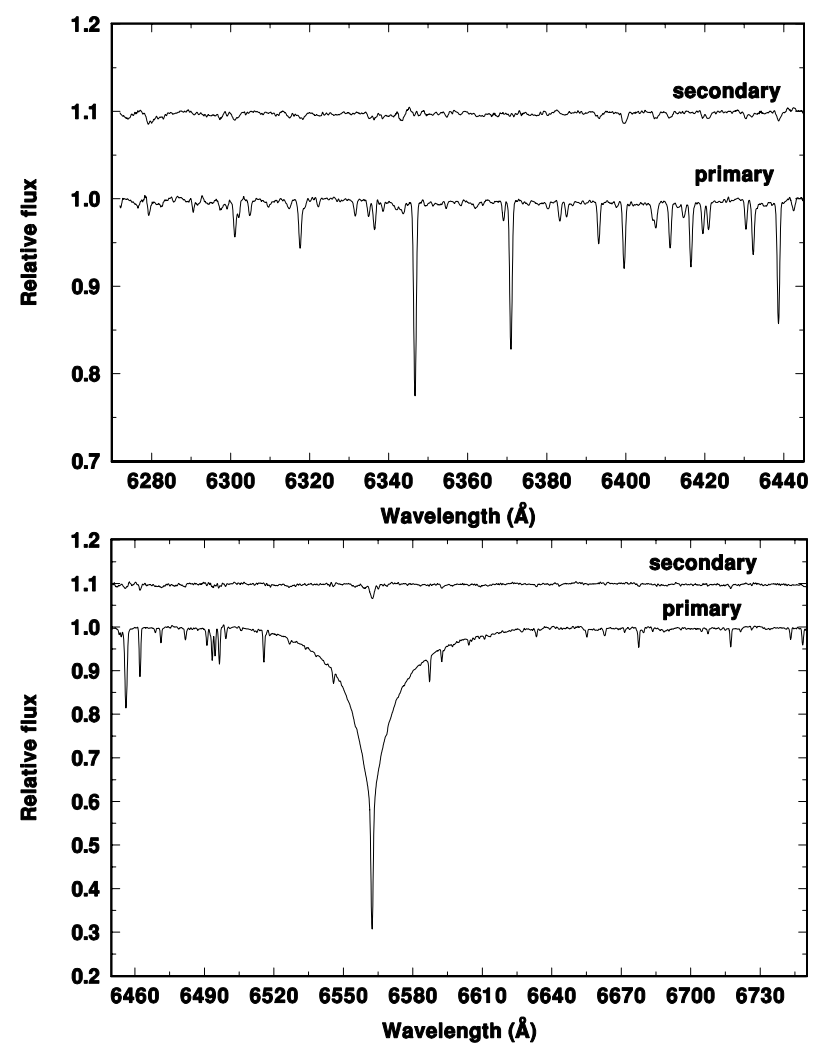

Fig. 12. KOREL-disentangled red spectra of the primary and secondary components of HD 143418 displayed in two wavelength segments. For clarity, the secondary spectrum was shifted for 0.1 in relative flux.

of ellipsoidal variability in a binary system. We therefore also decided to consider such a possibility quantitatively. For simultaneous analysis of photometric and spectroscopic (RV) data, we used PHOEBE 0.30 (Prša \& Zwitter 2005) based on the WD 2004 code (Wilson \& Devinney 1971).

\subsection{Ellipsoidal variability}

As demonstrated in Sect. 3, the amplitude and shape of the orbital light curve of HD 143418 are secularly variable. However, this variability is not as pronounced during the first four seasons (HJD 2445117-2449057), and also in season 8, as it is in the remaining ones. We thus assembled the four first seasons into a joint data set, each passband separately, removed $3 \sigma$ outliers, and derived a reference solution of the binary. Since there are several caveats in our analysis, we proceed with a step-by-step description.

To estimate the effective temperature of the binary as accurately as possible, several independent approaches were used ${ }^{3}$ :

1. via comparison between Kurucz's model atmospheres and the disentangled spectrum of the primary star, as explained in Sect. 4.1. This gave $T_{\text {eff }}=7700 \mathrm{~K} \pm 200 \mathrm{~K}$ for the effective temperature of the primary. It must be kept in mind, however, that the observed spectrum contains a small contribution from the secondary, which may affect the above estimate. Indeed, the KOREL disentangled spectrum of the primary can also be reconciled with $T_{\text {eff }}$ of some $8000 \mathrm{~K}$;

2. using Flower's (1996) corrected tables published by Prša \& Zwitter (2005). The mean color-index value for all seasons

3 See Prša \& Zwitter (2006a) for the concept of the effective temperature of the whole binary and its application to color-index constraints. 
is $B-V=0.16 \pm 0.01$, which yields the estimate $T_{\mathrm{eff}}=$ $8016 \mathrm{~K} \pm 66 \mathrm{~K}$

3. using Hauck \& Mermilliod (1998) mean values of Strömgren and $\mathrm{H} \beta$ photometric indices:

$b-y=0.094, m_{1}=0.189, c_{1}=0.981, \beta=2.854$.

These values, Hvar $V$ magnitude, and the program UVBYBETA $^{4}$ yield the dereddened magnitude of the binary $V_{0}=7.40$ and $E(b-y)=0$. 012 , which translates to $T_{\text {eff }}$ $=8000 \mathrm{~K} \pm 80 \mathrm{~K}$.

4. The mean all-sky magnitude and colors of HD 143418

$V=7^{\mathrm{m}} .451 \quad B-V=0^{\mathrm{m}} .159, \quad U-B=0^{\mathrm{m}} .117$,

correspond to an essentially unreddened A6 star. According to Lang (1999) this corresponds to $T_{\text {eff }} \sim 8000 \mathrm{~K}$.

From these results we adopt a conservative estimate of the effective temperature of the binary $T_{\text {eff }}=8000 \pm 300 \mathrm{~K}$. This value was used in the color-constraining method.

The main difficulty in modeling ellipsoidal binaries is to determine the luminosity ratio between the two components: in the case of eclipsing binaries, a luminosity ratio is revealed by the depth of the eclipses. In their absence, photometry is powerless for its determination. Figure 13 demonstrates this issue. For the given value of the $V$ passband luminosity ratio $L_{2}^{V} / L_{\text {tot }}^{V}$, the upper panel depicts a relation between the temperature and effective potentials (acting on behalf of stellar radii); this relation significantly deviates from the black-body approximation $L \propto R^{2} T^{4}$ because of the stellar atmosphere models and tidal and rotational distortion of the primary. The lower two panels depict the $\chi^{2}$ value of the fit as a function of $\Omega_{2} / \Omega_{1}$ (left) and $T_{2} / T_{1}$ (right). Due to significant data scatter in our observations, lower $\chi^{2}$ values (higher $L_{2}^{V} / L_{\text {tot }}^{V}$ values) do not imply a better solution in a physical sense, only in a sense of least squares ${ }^{5}$. The $\chi^{2}$ curve is flat for pretty much all luminosity ratios $L_{2}^{V} / L_{\text {tot }}^{V}<0.15$ over a broad range of $\Omega_{2} / \Omega_{1}$ and $T_{2} / T_{1}$ values of parameters. In particular, if we somehow knew that the luminosity ratio $L_{2}^{V} / L_{\text {tot }}^{V}=0.05$, any value of $T_{2} / T_{1}$ between 0.7 and 1.1, and the corresponding value of $\Omega_{2} / \Omega_{1}$ satisfying the upper panel relation, will yield an identical $\chi^{2}$ value. The two problems to solve are thus: 1 ) what is the approximate luminosity ratio $L_{2}^{V} / L_{\text {tot }}^{V}$, and 2) given the luminosity ratio, which pair of $\left(\Omega_{2} / \Omega_{1}, T_{2} / T_{1}\right)$ values is most appropriate?

The first problem can be resolved by spectroscopy: by disentangling a weak secondary star imprint in the spectra at different phases with KOREL, we estimate the $\mathrm{V}$ passband luminosity ratio $L_{2}^{V} / L_{\text {tot }}^{V}$ to be between 4 and 8 percent. Better estimates will only be possible when more spectra are available. For the time being, we adopt the luminosity ratio to be $L_{2}^{V} / L_{\text {tot }}^{V}=0.06 \pm 0.02$.

The second problem may be resolved by the parallax measurements published by Perryman \& ESA (1997): $\pi=$ $0{ }^{\prime} 00562 \pm 0.0^{\prime} 00062$. Luminosity ratio $L_{2}^{V} / L_{\text {tot }}^{V}=0.06 \pm 0.02$ implies that the primary star contribution is $V_{0}=7 \mathrm{~m}$. 47 . From this, the parallax, $T_{\text {eff }}=8000 \mathrm{~K}$, and a bolometric correction interpolated from Code et al. (1976), we obtained the following estimate for the primary star radius:

$R_{1}=2.65_{-0.27}^{+0.33} R_{\odot}$.

${ }^{4}$ Written by T.T. Moon at University London in 1985 and modified by R. Napiwotzki at Kiel University in 1992: (Napiwotzki, Priv. Com., based on the work by Moon \& Dworetsky 1985).

${ }^{5}$ Light curve that features eclipses happens to yield a better leastsquares value, although it is obvious that such a solution is implausible.

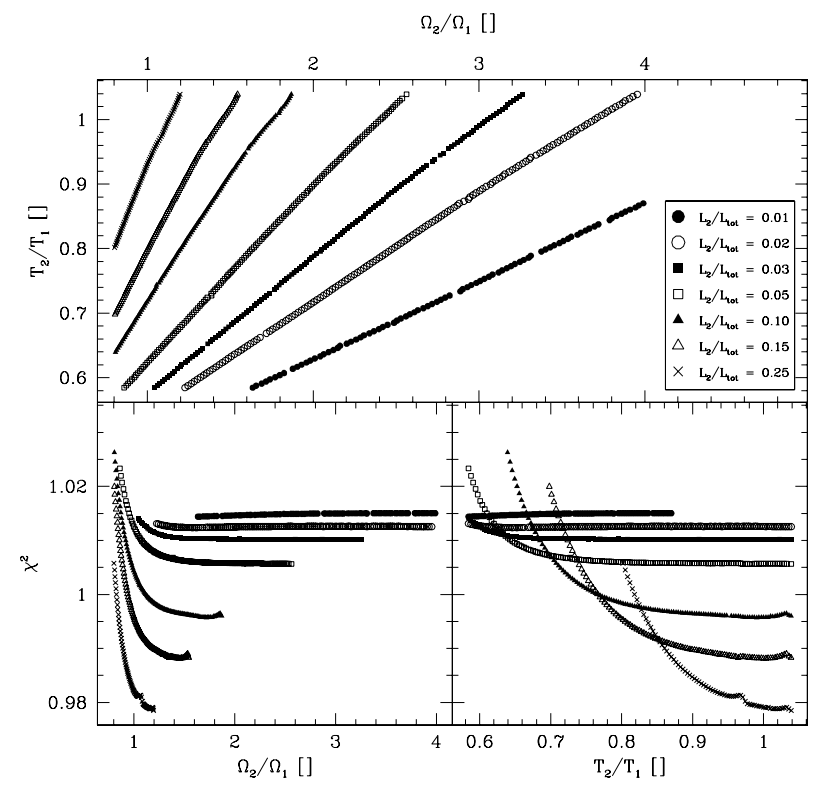

Fig. 13. The determination of the secondary-to-primary star luminosity ratio. In absence of eclipses, photometry cannot reveal this ratio. The upper panel shows the relation between the temperature ratio and the ratio of effective potentials (acting on behalf of stellar radii) for the given luminosity ratio; the lower two panels depict the $\chi^{2}$ dependence on $\Omega_{2} / \Omega_{1}$ (left) and $T_{2} / T_{1}$ (right). The flatness of the $\chi^{2}$ curve prevents us from pinpointing the luminosity ratio from photometry alone.

Adopting $K_{1}=80.2 \mathrm{~km} \mathrm{~s}^{-1}$ and $K_{2}=133.3 \mathrm{~km} \mathrm{~s}^{-1}$ from solution 2 of Table 6 , one obtains:

$M_{1} \sin ^{3} i=1.437 M_{\odot}, \quad M_{2} \sin ^{3} i=0.865 M_{\odot}$.

According to Harmanec (1988), a normal mass of the primary with its $T_{\text {eff }}=8000 \mathrm{~K}$ is $M_{1}=1.81 M_{\odot}$. For the nominal values above this implies an orbital inclination of $67.8^{\circ}$, companion mass $M_{2}=1.09 M_{\odot}$, and the binary separation $a=10.40 R_{\odot}$. The corresponding Roche-lobe radii are 4.40 and $3.49 R_{\odot}$. Note also that for the estimated range of the primary radius one obtains $\log g / g_{0}=3.849_{-0.102}^{+0.093}$ for the primary, in an acceptable agreement with the estimate based on the synthetic spectra. It is reasonable to assume that the secondary is essentially unevolved and its radius, corresponding to its estimated mass, and should be about $1.1 R_{\odot}$. This back-of-the-envelope estimate reassures us that the binary components are well detached, and the values of the effective potentials may be estimated to be $\Omega_{1}=4.56$ and $\Omega_{2}=6.89$. Consulting the upper panel of Fig. 13 for the ratios $\Omega_{2} / \Omega_{1}=1.51$ and $L_{2}^{V} / L_{\text {tot }}^{V}=0.06$ yields an estimate $T_{2} / T_{1}=0.80$. Finally, color constraining leads to individual temperatures of both stars: $T_{1}=8190 \mathrm{~K}$ and $T_{2}=6550 \mathrm{~K}$. With the values above, one can also estimate the minimum orbital inclination under which the stars would eclipse each other with respect to an observer on the Earth,

$\cos i_{\min }=\frac{R_{1}+R_{2}}{a}=68.9^{\circ}$.

This implies that HD 143418 is very close to a grazing eclipser.

\subsection{Subsynchronous rotation}

For a binary with a period of 2.28252 one would expect a spinorbit synchronization and also that the orbital plane and the equatorial plane of the primary are parallel. This, however, leads to the following problem. The observed $v \sin i=18 \mathrm{~km} \mathrm{~s}^{-1}$ and 
inclination $i=68^{\circ}$ would imply a primary radius as small as $0.9 R_{\odot}$, which contradicts both the spectral class and the parallax. It is easy to verify that this problem cannot be avoided over the whole range of plausible masses for an A star primary. Our estimate of the primary radius implies the rotational period of the primary between about 6.0 and 7.0 . Notably, some other $\mathrm{CP}$ stars in binaries were also found to have subsynchronous rotation: HgMn stars HR 266, HR 4072, $\chi$ Lup, and 74 Aqr, and metal weak A0III stars HR 7338 (see Dworetsky 1993 and references therein) or V624 Her = HR 6611 (Mikulášek et al. 2004). To the best of our knowledge, there is no plausible explanation of this dynamically unusual situation. Mikulášek et al. (2004) speculate about the possibility that the rotational axis of the subsynchronous primary of V624 Her is not perpendicular to the orbital plane of the binary. However, the whole effect is mild for V624 Her (4.3 vs. 3. 895 orbital period) in comparison to HD 143418.

\subsection{Computing the reference solution}

The above estimated values of parameters were used as an initial guess for thorough modeling with PHOEBE. The solution is depicted in Fig. 14 and the corresponding elements are listed in Table 7. HD 143418 may indeed be viewed as an ellipsoidal variable with a subsynchronous, slightly distorted primary $\left(R_{\text {pole }} / R_{\text {point }}=0.98\right)$.

In the first step we fitted the observed RV curves to obtain parameters that are predominantly sensitive to this type of data, namely $a \sin i, q=M_{2} / M_{1}$, and $v_{\gamma}$ (cf. Table 7$)^{6}$. These values, once obtained, were kept fixed throughout subsequent analysis. Then, keeping the $V$ passband luminosity fixed, we proceeded to improve our initial estimate by heuristic scanning with Powell's method (Prša \& Zwitter 2006b). The model seems to prefer a slightly lower inclination, $i \sim 65^{\circ}$ instead of $68^{\circ}$, but the error estimates easily allow perfect reconciliation. The final reference solution for seasons 1-4 is given in Table 7. Taken at face value, it implies the rotational period of the primary of $6.8 \pm 0.2$.

\subsection{Comparison with evolutionary models}

The reference solution enables us to compare the properties of both components with the evolutionary tracks from Schaller et al. (1992) evolutionary models. Figure 15 depicts the positions of the primary and secondary in the $\log R$ vs. $\log T_{\text {eff }}$ diagram. One can see that both binary components fall onto evolutionary tracks of stars of somewhat higher masses than the masses which follow from the reference solution. However, the discrepancy is not large and remains within the estimated errors of the elements involved. As assumed in Sect. 5.1, the secondary is an unevolved main-sequence object.

\subsection{Towards the model of secular variations}

It remains to be seen whether secular variations in light curves of seasons 5-8 may be plausibly explained by an adequate physical model. The subsynchronous rotation of the primary a priori prohibits the existence of a cool spot on its surface, since phase curves in all seasons show a remarkable coherence. Whatever

\footnotetext{
${ }^{6}$ To obtain $a \sin i$, a standard "trick" is used: the inclination is set at a value of $i=90^{\circ}$ and $a$ is fitted; the value thus obtained is then actually $a \sin i$. It is then kept constant for subsequent minimizer runs, in which allowance for convergency of inclination $i$ can be made, by PHOEBE's conditional constraining method.
}

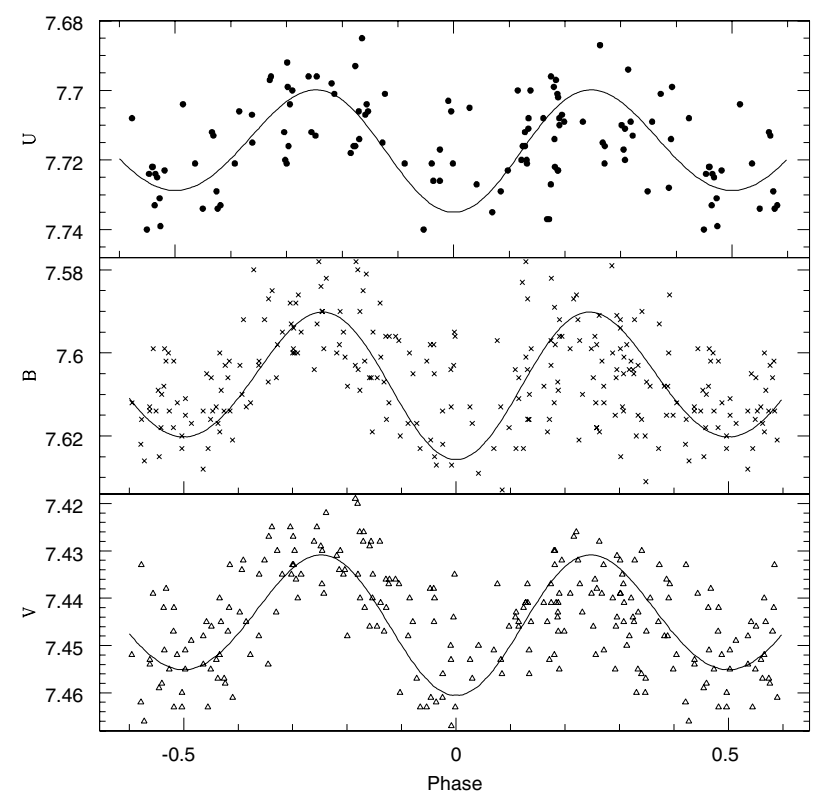

Fig. 14. Light-curve solution for seasons $1-4$. The variability can completely be attributed to ellipsoidal variations of the primary; the corresponding elements are given in Table 7. This solution is taken as the reference one for the determination of secular light changes seen in the other seasons.

Table 7. Physical and geometrical parameters of the reference solution depicted in Fig. 14. The epoch of superior conjunction of the primary is in HJD-2 449 100. Parameter errors given in the table are formal, obtained from the covariance matrix.

\begin{tabular}{|c|c|c|c|}
\hline \multirow[t]{2}{*}{ Parameter: } & \multicolumn{3}{|c|}{ System: } \\
\hline & Primary: & & Secondary: \\
\hline$P$ & & 2.282520 fixed & \\
\hline$T_{\text {super.conj. }}$ & & $78.43247 \pm 0.00099$ & \\
\hline$a\left[R_{\odot}\right]$ & & $10.4 \pm 0.4$ & \\
\hline$i\left[^{\circ}\right]$ & & $65.3 \pm 4.9$ & \\
\hline$q=M_{2} / M_{1}$ & & $0.62 \pm 0.02$ & \\
\hline$v_{\gamma}\left[\mathrm{km} \mathrm{s}^{-1}\right]$ & & $-22.0 \pm 0.8$ & \\
\hline$T_{\text {eff }}[\mathrm{K}]$ & $8200 \pm 123$ & & $6400 \pm 75$ \\
\hline $\log g / g_{0}$ & $3.88 \pm 0.58$ & & $4.41 \pm 0.66$ \\
\hline$\Omega^{a}$ & $4.60 \pm 0.09$ & & $6.95 \pm 0.16$ \\
\hline$F^{b}$ & 0.35 & & 1.0 \\
\hline$A^{b}$ & 0.6 & & 0.6 \\
\hline$\beta^{c}$ & 1.0 & & 0.32 \\
\hline$M\left[M_{\odot}\right]$ & $1.79 \pm 0.21$ & & $1.11 \pm 0.13$ \\
\hline$R\left[R_{\odot}\right]$ & $2.67 \pm 0.06$ & & $1.10 \pm 0.03$ \\
\hline$L\left[L_{\odot}\right]$ & $28.1 \pm 0.29$ & & $1.89 \pm 0.11$ \\
\hline$R_{\text {pole }}\left[R_{\odot}\right]$ & $2.60 \pm 0.11$ & & $1.12 \pm 0.05$ \\
\hline$R_{\text {point }}\left[R_{\odot}\right]$ & $2.65 \pm 0.12$ & & $1.12 \pm 0.05$ \\
\hline$R_{\text {side }}\left[R_{\odot}\right]$ & $2.61 \pm 0.11$ & & $1.12 \pm 0.05$ \\
\hline$R_{\text {back }}\left[R_{\odot}\right]$ & $2.64 \pm 0.12$ & & $1.12 \pm 0.05$ \\
\hline$x_{\mathrm{LD}, U}^{d}$ & 0.710 & & 0.847 \\
\hline$x_{\mathrm{LD}, B}{ }^{d}$ & 0.809 & & 0.811 \\
\hline$x_{\mathrm{LD}, V}^{d}$ & 0.697 & & 0.718 \\
\hline$y_{\mathrm{LD}, U}{ }^{d}$ & 0.259 & & 0.235 \\
\hline$y_{\mathrm{LD}, B}{ }^{d}$ & 0.328 & & 0.228 \\
\hline$y_{\mathrm{LD}, V}{ }^{d}$ & 0.288 & & 0.275 \\
\hline
\end{tabular}

${ }^{a}$ Kopal potentials as defined by Wilson (1979).

${ }^{b}$ Albedo and synchronicity parameter: values are assumed and kept fixed.

${ }^{c}$ Gravity darkening coefficient: 1.0 after Von Zeipel (1924) and 0.32 after Lucy (1967).

${ }^{d}$ Logarithmic limb darkening coefficients taken from van Hamme (1993). 


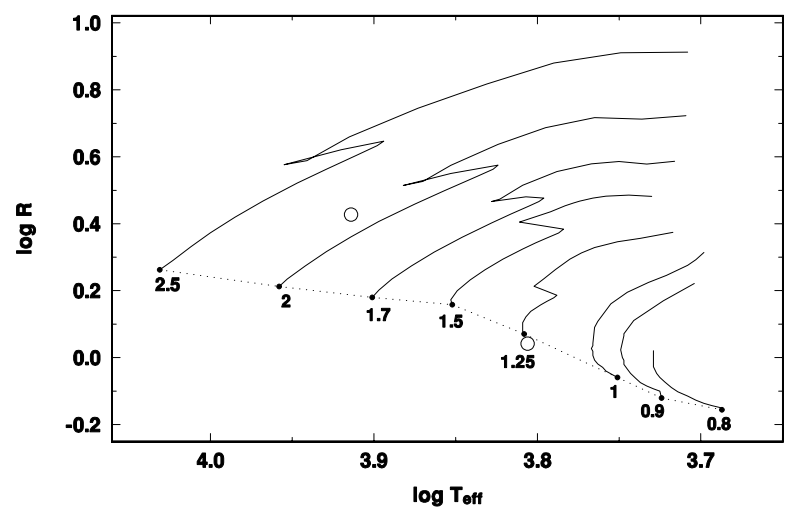

Fig. 15. The position of the primary and secondary of HD 143418 according to the reference solution (large open circles) in the $\log R$ vs. $\log T_{\text {eff }}$ diagram based on the evolutionary models of Schaller et al. (1992). The masses of model stars are given for all individual evolutionary tracks. The zero-age main sequence is shown by a dotted line.

effect is causing secular variations, it is almost certainly phaselocked to the orbital period.

We can think of two physical scenarios that could plausibly explain the observed secular variation: 1) a localized ejection on the primary and 2) a cool, large spot on the secondary. An ejection on the primary, in contrast to a surface spot, is not phaselocked to the revolution of the star around its axis, nor is it in general locked to any other systemic frequency: it depends on the direction of the ejection with respect to the center-of-mass of the system. However, since the tangential velocity of the primary at the equator is roughly $4 \times$ smaller than the velocity of the star itself around the center of mass, in most cases the ejection would pick up a strongest dynamical contribution - the orbital frequency. This would be the most prominent should an ejection occur close to the direction opposite from the center-of-mass of the system. It is thus quite possible to have an imprint on the light curves that would be phase-locked to the orbital period.

The second scenario, a cool spot on the secondary, benefits from an intuitive interpretation: since the secondary is assumed to co-rotate, a surface spot would be inevitably phase-locked to the orbital period. However, since the contribution of the secondary to the total system flux is only about 6 percent, the spot has to be very large (effectively covering the whole stellar hemisphere in case of season 5) and very cool (roughly 40 percent cooler than the underlying surface). If the F-type secondary is indeed rotating rapidly due to its spin-orbit synchronization, it can be chromospherically quite active. The chemical peculiarity of the primary may also indicate presence of magnetic fields and these could be responsible for both phenomena.

Regrettably, we do not have any spectra from the time interval around HJD 2451372-2452477 where the most peculiar light curves were observed. This renders our subsequent analysis a somewhat speculative one. In order not to over-interpret the data, we limit ourselves to a simple description of a single, circularlyshaped prominence, be it a localized ejection or a spot. Both may be described by 4 parameters: stellar latitude $\theta_{\mathrm{s}}$ and longitude $\phi_{\mathrm{s}}$, angular radius $\rho_{\mathrm{s}}$ given in radians, and the temperature factor $\tau_{\mathrm{s}}=T_{\text {spot }} / T_{\text {star. }}$. Due to data scatter, a traditional modeling approach with differential corrections (DC) turned out to be unstable. We therefore replaced DC with a Monte Carlo algorithm over a wide range of plausible parameter values. Figure 16 shows an example of the $\tau_{\mathrm{s}}-\rho_{\mathrm{s}}$ cross-section for the ejection (left) and a spot (right). The degeneracy is particularly accute in the case
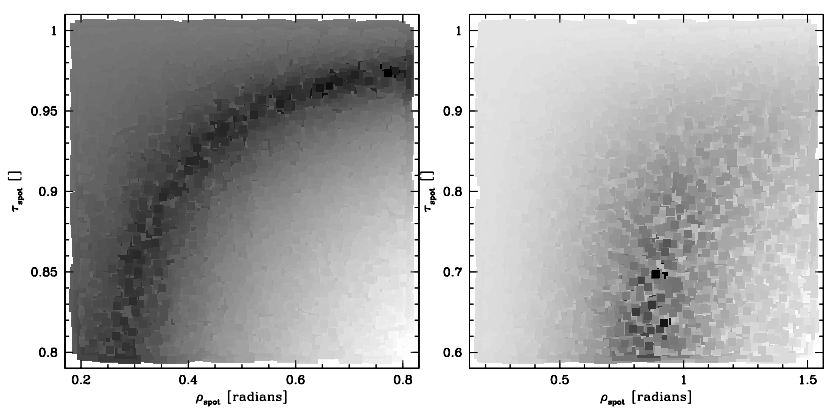

Fig. 16. $\chi^{2}$ values of the $\tau_{\text {spot }}-\rho_{\text {spot }}$ cross-section. Shades of gray are determined by the Monte Carlo simulation and they correspond to $\chi^{2}$ values of the fit: dark colors signify low $\chi^{2} \mathrm{~s}$ and thus better mat ch with observations. The left panel depicts a cross-section for the localized ejection model on the primary; a strong degeneracy between $\rho_{\text {spot }}$ and $\tau_{\text {spot }}$ is evident. The right panel depicts a cross-section for the spot model on the secondary; the degeneracy is less significant, but still present.

Table 8. Table of parameters for the localized ejection scenario (left) and a spot scenario (right) with their respective formal errors. An asterisk in the table means that the value has been tentatively set and kept fixed throughout the analysis.

\begin{tabular}{cccc}
\hline \hline Season: & Parameter: & Ejection: & Spot: \\
\hline \multirow{3}{*}{5} & $\theta_{\mathrm{s}}$ & $1.57^{*}$ & $1.57^{*}$ \\
& $\phi_{\mathrm{s}}$ & $5.14 \pm 0.05$ & $1.97 \pm 0.06$ \\
& $\rho_{\mathrm{s}}$ & $0.77 \pm 0.05$ & $1.23 \pm 0.09$ \\
& $\tau_{\mathrm{s}}$ & $0.974 \pm 0.004$ & $0.61 \pm 0.11$ \\
\hline \multirow{3}{*}{6} & $\theta_{\mathrm{s}}$ & $1.57^{*}$ & $1.57^{*}$ \\
& $\phi_{\mathrm{s}}$ & $5.25 \pm 0.10$ & $2.09 \pm 0.28$ \\
& $\rho_{\mathrm{s}}$ & $0.62 \pm 0.17$ & $0.89 \pm 0.32$ \\
& $\tau_{\mathrm{s}}$ & $0.974 \pm 0.009$ & $0.70 \pm 0.16$ \\
\hline \multirow{3}{*}{7} & $\theta_{\mathrm{s}}$ & $1.57^{*}$ & $1.57^{*}$ \\
& $\phi_{\mathrm{s}}$ & $4.90 \pm 0.15$ & $1.71 \pm 0.14$ \\
& $\rho_{\mathrm{s}}$ & $0.44 \pm 0.15$ & $0.61 \pm 0.13$ \\
& $\tau_{\mathrm{s}}$ & $0.974 \pm 0.013$ & $0.68 \pm 0.14$ \\
\hline \multirow{2}{*}{8} & $\theta_{\mathrm{s}}$ & $1.57^{*}$ & $1.57^{*}$ \\
& $\phi_{\mathrm{s}}$ & $5.16 \pm 0.28$ & $2.01 \pm 0.28$ \\
& $\rho_{\mathrm{s}}$ & $0.24 \pm 0.14$ & $0.33 \pm 0.11$ \\
& $\tau_{\mathrm{s}}$ & $0.974 \pm 0.028$ & $0.62 \pm 0.22$ \\
\hline
\end{tabular}

of ejection: different combinations of $\left(\tau_{\mathrm{s}}, \rho_{\mathrm{s}}\right)$ match the observations equally well. Since the phase coherence of the light curve is very pronounced and the location of the prominence may be confined to a narrow phase interval, it is reasonable to assume that the cause of the secular variation originates at the equatorial parts of the primary. Taking this into account, we tentatively set the latitude of the prominence at the equator $\left(\theta_{\mathrm{s}}=\pi / 2\right)$ and keep it fixed throughout the analysis.

In the case of ejection we cannot resolve the degeneracy in $\tau_{\mathrm{s}}-\rho_{\mathrm{s}}$ in any thorough-going manner (c.f. Fig. 16, left panel). For the sake of the simplicity of the physical description, we assume that the temperature factor $\tau_{\mathrm{s}}$ does not change much from season to season and that most of the variability can be attributed to the decaying size of the prominence. Table 8 (left) summarizes the parameters of such an ejection scenario for seasons 5-8, while the comparison of its model prediction with the observed $V$-band light curves is shown in the left panels of Fig. 17. Notably, the longitude of the ejection remains virtually unchanged for all seasons, and its angular size diminishes monotonically with time. 


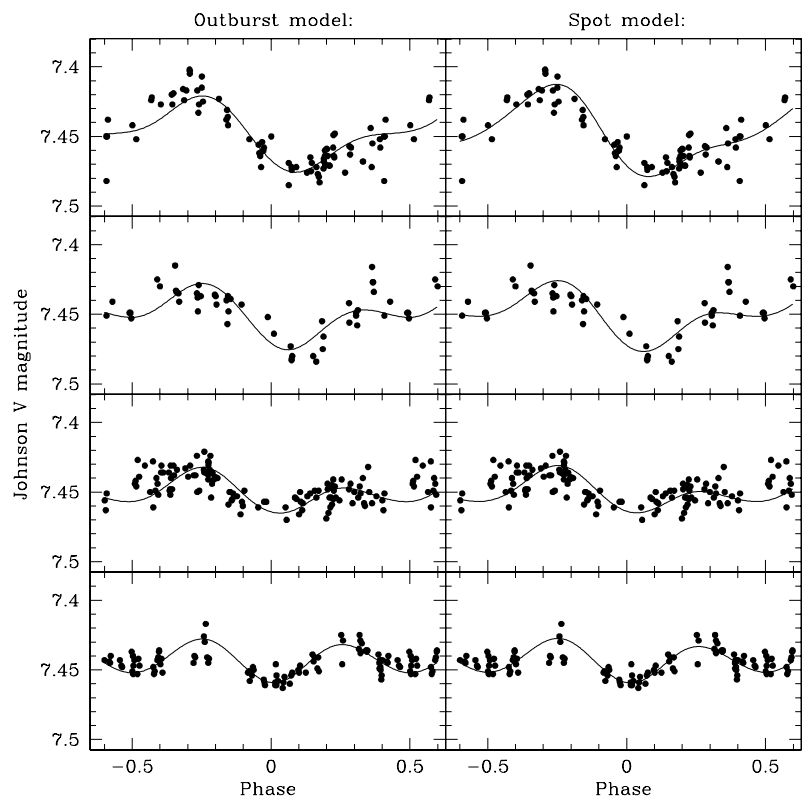

Fig. 17. A comparison of the two alternative models of secular variations with the observed $V$-band light curves (represented by individual data points). The panels on the left show the solution for the localized ejection model (cf. Table 8, left), those on the right for the spot model (cf. Table 8, right) for seasons 5-8. In terms of the $\chi^{2}$ value, no discrimination between the two alternatives is possible.

However, the hypothesis of a spot on the secondary satisfies the observations equally well - see the right column of Table 8 and Fig. 17. It might be difficult to envision a mechanism that would produce a spot so large and so cool as is featured in season 5, but we note again that the primary of HD 143418 is chemically peculiar and the presence of strong magnetic fields in the system is quite plausible. Furthermore, the spot solution does not suffer from the degeneracy as much as the solution for the ejection, which allows us to derive parameters formally, without any assumptions. We again see the positional constancy, the unchanged temperature factor $\tau_{\mathrm{s}}$, and the diminishing angular size $\rho_{\mathrm{s}}$ of the spot from season to season. This remarkable result allows the speculation that, no matter what the origin of the prominence is, we are observing its appearence and sequential dissapearence. Perhaps too boldly, we speculate that the dynamical time scale derived from the last 4 seasons might be typical for such prominences on other chemically peculiar stars as well.

A quantitative modeling of the secular changes allows another important conclusion to be made: it is an independent confirmation of the subsynchronous rotation of the primary. Figure 18 depicts the prominence in season 5 computed for different values of synchronicity parameters $F_{1}=0.35$ to $F_{1}=2.5$. The slope of the light curve clearly prefers models with low $F_{1}$, surely not over 1 and very probably significantly less. The same conclusion invariably follows from all passbands and all seasons, so we discard the suspicion that this may be an artifact of noisy data.

\subsection{The value of the orbital period}

According to Gerbaldi et al. (1985) and Guthrie (1986), there is a lack of orbital periods shorter than about 3 days among $\mathrm{CP}$ stars in binaries. Only recently, a discovery of the SB1 binary HD 200405 with an orbital period of 1.635 was reported by Carrier et al. (2002). Our discovery of the duplicity

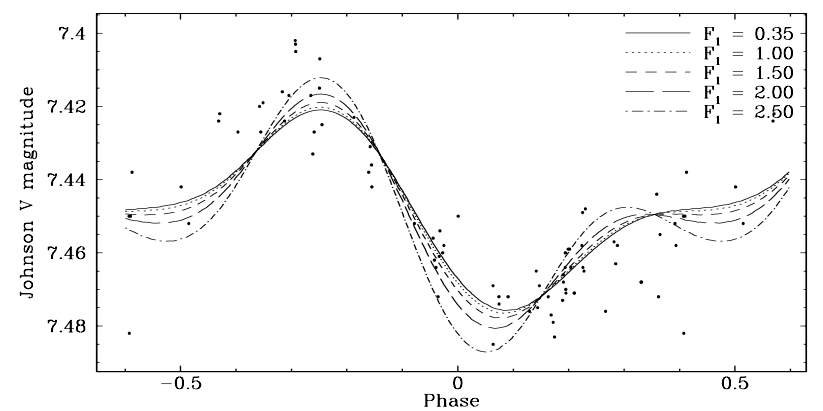

Fig. 18. Influence of the synchronicity parameter value $F$ on season 5 Johnson $V$ light curve. Observations are denoted with points, synthetic curves are drawn with different line types given in the legend above. Each solution corresponds to the different value of $F$, while all remaining parameters are kept unchanged. By inspecting the shape of the light curve (especially its slope on the phase interval -0.25-0.0), it is evident that the solutions with low, subsynchronous values of $F$ are preferable. The significance of this effect is due to rotational deformation of the already distorted primary and thus a measurable change in the surface cross-section. Other seasons and other passbands re-confirm this finding but for brevity we omit them here.

of HD 143418 may, therefore, contribute to a better understanding of the short-period deficiency among CP stars.

Acknowledgements. We thank Drs. Z. Mikulášek and J. Zverko, and the referee, Dr. P. North, for helpful comments and for constructive criticism of earlier versions of this manuscript. We profited from the use of the bibliographic services of CDS in Strasbourg and ADS of NASA. We acknowledge the use of the programs FOTEL and KOREL written by Dr. P. Hadrava and UVBYBETA written by Drs. T. T. Moon and R. Napiwotzki. Dr. J. Kubát kindly provided a grid of synthetic spectra calculated with SYNSPEC. The Czech authors were supported by the research plans J13/98: 113200004 of Ministry of Education, Youth and Sports and AV 0Z1 003909, from project K2043105 of the Academy of Sciences of the Czech Republic and from the grants GA ČR 205/03/0788 and 205/06/0304 of the Czech Science Foundation. UBV observations at the SPM Observatory were secured in the course of the Czech-Mexican KONTAKT ME402(2000)/CONACyT E130.734 collaborative project. The collaboration between Turkish and Czech authors was facilitated thanks to a NATO collaborative linkage grant PST.CLG.979343. The mutual collaborative visits between Czech and Slovenian coauthors were facilitated thanks to project 22/2006-2007 of the Czech-Slovenian Intergovernmental Cooperation Programme.

\section{References}

Adelman, S. J. 1998, A\&AS, 132, 93

Alania, I. F., Abuladze, O. P., \& West, R. M. 1989, A\&AS, 77, 333

Bartaya, R. A. 1979, Abastuman. Astrofiz. Obs. Bull., 51, 1

Carrier, F., North, P., Udry, S., \& Babel, J. 2002, A\&A, 394, 151

Code, A. D., Bless, R. C., Davis, J., \& Brown, R. H. 1976, ApJ, 203, 417

Deeming, T. J. 1975, Ap\&SS, 36, 137

Dworetsky, M. M. 1993, in IAU Colloq. 138: Peculiar versus Normal Phenomena in A-type and Related Stars, ed. M. M. Dworetsky, F. Castelli, \& R. Faraggiana, ASP Conf. Ser., 44, 1

Flower, P. J. 1996, ApJ, 469, 355

Gerbaldi, M., Floquet, M., \& Hauck, B. 1985, A\&A, 146, 341

Guthrie, B. N. G. 1986, MNRAS, 220, 559

Hadrava, P. 1990, Contributions of the Astronomical Observatory Skalnate Pleso, 20, 23

Hadrava, P. 1995, A\&AS, 114, 393

Hadrava, P. 1997, A\&AS, 122, 581

Hadrava, P. 2004a, Publ. Astron. Inst. Czech. Acad. Sci., 92, 1

Hadrava, P. 2004b, Publ. Astron. Inst. Czech. Acad. Sci., 92, 15

Harmanec, P. 1988, Bull. Astron. Inst. Czech., 39, 329

Harmanec, P. 1998, A\&A, 335, 173

Harmanec, P., \& Horn, J. 1998, J. Astron. Data, 4, 5

Harmanec, P., Horn, J., Koubský, P., et al. 1980, Bull. Astron. Inst. Czech., 31, 144

Harmanec, P., Horn, J., \& Koubský, P. 1982, in Be Stars, ed. M. Jaschek \& H.-G. Groth, IAU Symp., 98, 269

Harmanec, P., Matthews, J. M., Božić, H., Pavlovski, K., \& Huang, L. 1991, Bull. Astron. Inst. Czech., 42, 1 
Harmanec, P., Horn, J., \& Juza, K. 1994, A\&AS, 104, 121

Harmanec, P., Hadrava, P., Yang, S., et al. 1997a, A\&A, 319, 867

Harmanec, P., Pavlovski, K., Božić, H., et al. 1997b, J. Astron. Data, 3, 5

Hauck, B., \& Mermilliod, M. 1998, A\&AS, 129, 431

Horn, J., Kubát, J., Harmanec, P., et al. 1996, A\&A, 309, 521

Hubeny, I., Štefl, S., \& Harmanec, P. 1985, Bull. Astron. Inst. Czech., 36, 214

Kurucz, R. 1993, Atomic data for opacity calculations. Kurucz CD-ROM No. 1. Cambridge, Mass.: Smithsonian Astrophysical Observatory, 1993, 1

Kurucz, R. 1994, Solar abundance model atmospheres for 0,1,2,4,8 $\mathrm{km} \mathrm{s}^{-1}$. Kurucz CD-ROM No. 19. Cambridge, Mass.: Smithsonian Astrophysical Observatory, 1994, 19

Lang, K. R. 1999, Astrophysical formulae (Astrophysical formulae / K. R. Lang. New York : Springer, Astronomy and astrophysics library)

Lenz, P., \& Breger, M. 2005, Commun. Asteroseismol., 146, 53

Lucy, L. B. 1967, Z. Astrophys., 65, 89

Manfroid, J., \& Renson, P. 1989, A\&A, 223, 187

Mikulášek, Z., Zverko, J., Romanyuk, I. I., et al. 2004, in Magnetic stars, Proceedings of the International Conference, held in the Special Astrophysical Observatory of the Russian AS, August 27-31, 2003, ed. Y. V. Glagolevskij, D. O. Kudryavtsev, \& I. I. Romanyuk, Nizhnij Arkhyz, 191

Moon, T. T., \& Dworetsky, M. M. 1985, MNRAS, 217, 305

Pavlovski, K., Harmanec, P., Božić, H., et al. 1997, A\&AS, 125, 75

Percy, J. R., \& Attard, A. 1992, PASP, 104, 1160

Percy, J. R., Harlow, J., Hayhoe, K. A. W., et al. 1997, PASP, 109, 1215
Perryman, M. A. C., \& ESA. 1997, The HIPPARCOS and TYCHO catalogues. Astrometric and photometric star catalogues derived from the ESA HIPPARCOS Space Astrometry Mission, The Hipparcos and Tycho catalogues. Astrometric and photometric star catalogues derived from the ESA Hipparcos Space Astrometry Mission, Publisher: Noordwijk, Netherlands: ESA Publications Division, Series: ESA SP Ser., 1200

Prša, A., \& Zwitter, T. 2005, ApJ, 628, 426

Prša, A., \& Zwitter, T. 2006a, ArXiv Astrophysics e-prints

Prša, A., \& Zwitter, T. 2006b, ArXiv Astrophysics e-prints

Rakosch, K. D., \& Fiedler, W. 1978, A\&AS, 31, 83

Schaller, G., Schaerer, D., Meynet, G., \& Maeder, A. 1992, A\&AS, 96, 269

Schuster, W. J., Parrao, L., Gonazlez-Bedolla, S. F., Rios-Herrera, M., \& Berumen, M. R. 1985, Rev. Mex. Astron. Astrofis., 11, 55

Škoda, P. 1996, in Astronomical Data Analysis Software and Systems V, ed. G. H. Jacoby \& J. Barnes, ASP Conf. Ser., 101, 187

Stagg, C. R., Božić, H., Fullerton, A. W., et al. 1988, MNRAS, 234, 1021 van Hamme, W. 1993, AJ, 106, 2096

Vidal, C. R., Cooper, J., \& Smith, E. W. 1973, ApJS, 25, 37

von Zeipel, H. 1924, MNRAS, 84, 665

Wilson, R. E. 1979, ApJ, 234, 1054

Wilson, R. E., \& Devinney, E. J. 1971, ApJ, 166, 605

Winzer, J. 1974, Ph.D. Thesis, University of Toronto

Zboril, M., \& Budaj, J. 1999, Inf. Bull. Variable Stars, 4748, 1 